\title{
Effect of anti-gut inflammatory agent on insulin resistance and lipid profile of mice fed different diets
}

\author{
Zheng Wang ${ }^{1,2}$ and Zhijun Bao ${ }^{1 *}$ \\ ${ }^{1}$ Department of Geriatrics and Gastroenterology, Huadong Hospital, Shanghai Medical College, Fudan University, Shanghai \\ Key Laboratory of Clinical Geriatric Medicine, Shanghai 200040, PR China, ${ }^{2}$ University of Groningen, University Medical Center \\ Groningen, Department of Pediatrics, Section Molecular Genetics, Groningen 9713AV, The Netherlands
}

*For correspondence: Email: xinyi8681@sina.cn; Tel: (86)021-62483180

Sent for review: 21 September 2017

Revised accepted: 31 October 2017

\begin{abstract}
Purpose: To further explore the effect of 5-aminosalicylic acid (5-ASA) treatment on lipid levels in mice fed different diets.

Methods: Groups of 9 - 10 mice each were randomly assigned to 6 different diets, low-fat diet (LFD) with or without 5-ASA, high-fat diet (HFD) with or without 5-ASA, and high-fat high-cholesterol diet (HFC) with or without 5-ASA for 12 weeks.

Results: There were changes in gut microbiota of 5-ASA-treated mice, although gut permeability was similar between treated and non-treated groups. The level of fasting blood glucose, fasting plasma insulin and the curve of glucose tolerance test (GTT) in mice fed LFD, HFD or HFC diet were not affected by 5-ASA treatment. Although plasma lipid levels were similar between 5-ASA consuming and non-5-ASA groups in mice fed LFD and HFD, improved lipid profile was seen in mice that received $H F C+5-A S A$ when compared with mice fed only HFC.

Conclusion: These results indicate that targeting gut inflammation and dysbiosis with 5-ASA neither improves gut barrier nor insulin resistance (IR). Thus, results from therapies for metabolic disorder based on anti-gut inflammation should be interpreted with caution.
\end{abstract}

Keywords: 5-Aminosalicylic acid, Small intestine, High-fat, High-cholesterol diet, Dyslipidemia, Glucose tolerance

Tropical Journal of Pharmaceutical Research is indexed by Science Citation Index (SciSearch), Scopus, International Pharmaceutical Abstract, Chemical Abstracts, Embase, Index Copernicus, EBSCO, African Index Medicus, JournalSeek, Journal Citation Reports/Science Edition, Directory of Open Access Journals (DOAJ), African Journal Online, Bioline International, Open-J-Gate and Pharmacy Abstracts

\section{INTRODUCTION}

Obesity is emerging as one of the most harmful global public health problem. The disease is associated with a series of metabolic complications, not only including insulin resistance (IR), but also type 2 diabetes (T2DM), dyslipidemia. Numerous studies show that systemic inflammatory reaction has a vital role in the early stage of obesity and promotes obesityassociated complications [1-3]. Therefore, antiinflammatory scheme becomes as new treatments for metabolic disorders [4]. Although many preclinical studies support this opinor, results generated from clinical trials are quite controversial and still ongoing $[5,6]$.

Several conditions lead to systemic inflammation, and in recent years, gut inflammation and gastrointestinal flora have attracted more attention than ever before due to their relationship with the immune system and IR. High fat diet (HFD) is reported to induce lowgrade intestinal inflammation and dysbiosis of gut microbiota [7,8]. Intestinal inflammation and dysbiosis are believed to be related to broken and dysfunction of the intestinal wall, which may activate and further prompt systemic IR. 
Recently, a mice study suggested that targeting gut inflammation with 5-aminosalicylic acid (5ASA) reversed intestinal inflammatory response and improved systemic IR [9], but no data on lipid profile was presented [9]. 5-Aminosalicylic acid (5-ASA) is a notable agent which exert its anti-inflammation function mainly in the gut. Since metabolic abnormalities are strongly related to each other and are considered as independent risk factors to each other, the improvement of IR by 5-ASA treatment suggests that lipid levels may be affected as well. Moreover, taking non-steroidal anti-inflammatory drugs (NSAIDs) show benefits in hyperlipidemia, steatohepatitis and atherosclerosis [11]. In addition, cholesterol component of HFD is proved to be a potent intestinal inflammatory trigger [12].

The aim of the present study was to determining the role of intestinal inflammation in the pathogenesis and progression of diet-induced metabolic complications by studying metabolic parameters in mice fed low fat diet (LFD) with or without 5-ASA, high-fat diet (HFD) with or without 5-ASA, high-fat, high-cholesterol diet (HFC) with or without 5-ASA.

Although there are many studies that have targeted inflammation for the treatment of diabetes, the extent to which gut inflammation contributes to IR and dyslipidemia is still unclear, especially in mice challenged with high-fat, highcholesterol diet. To get more insight into this question in the present study, mice experiment was set up using the same diet composition as in the study by Luck et al. did [9]. C57BL/6J mice were then used in our experiments, randomly assigned 6 groups of mice to LFD or HFD or HFC with or without 5-ASA supplementation at $1500 \mathrm{mg} / \mathrm{kg} /$ day for 12 weeks. Some IR-related metabolic parameters were then assayed.

\section{EXPERIMENTAL}

\section{Mice and treatment}

The mice experimental protocols were approved by the University of Groningen, the Ethical Committee for Animal Experiments (approval no. 15246.2). And all experiments were carried out in accordance with the principles and guidelines generated and accepted by the European Convention which was aimed to protect and reduced the pain of Laboratory Animals [10]. Nine to ten male C57BL/6J mice, aged 10-12 weeks, were considered as one group, and each mice was kept individually in the Individual ventilated cages and maintained at $23{ }^{\circ} \mathrm{C}, 50 \%$ humidity, and $12 \mathrm{~h}$ light/12h dark cycle.
All the mice were able to access to food and water freely. They were randomly assigned to 6 groups, each having 10 mice, and fed either lowfat diet (LFD; the diet supplier is USA Research Diets, $10 \%$ of energy coming from fat), LFD + 5ASA, high-fat diet (HFD; the diet supplier is USA Research Diets, $60 \%$ of energy coming from fat ), HFD+5-ASA, high-fat high-cholesterol diet (HFC; the diet supplier is USA Research Diets, $60 \%$ of energy coming from fat and plus $0.25 \%$ cholesterol) or $\mathrm{HFC}+5-\mathrm{ASA}$ for 12 weeks. 5-ASA powder (Sigma-Aldrich, USA) was mixed homogenously with LFD, HFD and HFC diets as appropriate, and presented at 1,500 mg/kg/day.

\section{Metabolic studies and tissue preparation}

Body weight as well as food intake were documented before diet interventions and recorded weekly after diet interventions were started. At the end of 12-weeks diet intervention, fat or lean mass was analyzed (the machine model is Bruker Minispec Whole Body Composition Analyzer). Fresh fecal samples were collected before treatment and after 3 weeks of diet intervention to conduct gut microbiome sequencing [9] .

Glucose tolerance test (GTT) was carried at week 11 with a $2 \mathrm{~g} / \mathrm{kg}$ glucose i.p. injection [13]. Blood samples from the end of mice tail were collected in EDTA-coated tubes before and at 15, 30, 60, 90, 120 minutes after i.p. injection. Samples were spun at 3000 rpm for $10 \mathrm{~min}$ at 4 ${ }^{\circ} \mathrm{C}$ and insulin concentrations were measured in plasma using ELISA kit (Alpco Diagnositics, Salem, NH).

\section{Histological studies}

The biggest lope of liver from each mouse was taken and fixed in $4 \%$ phosphate-buffered formalin overnight, then samples were embedded in liquid paraffin overnight. For microscopic examination, liver sample were sectioned by Leica paraffin slicing machine at $4 \mu \mathrm{m}$ and hematoxylin and eosin (H\&E) was used for staining. Representative photomicrographs were captured at $100 \times$ magnification using the software incorporated in the microscope (Leica DM 3000).

\section{Quantification of plasma and liver lipids}

Triglycerides and cholesterol levels of plasma or liver were determined by Roche kits (Roche; Germany), and free cholesterol was measured by DiaSys kit (FS DiaSys, Holzhein, Germany) [14].

Trop J Pharm Res, November 2017; 16(11): 2652 


\section{HPLC sample preparation}

Plasma 5-ASA and $\mathrm{N}$-acetyl 5ASA were measured on a Waters HPLC system and Waters Empower software, consisting of a Waters 600 HPLC Pump, the model number is Waters 770 autosampler, Nova-Pak RP-C18 column and a Waters 717 Fluorescence Detector (the detector setting is $317 \mathrm{~nm}$ excitation, and for emission cut off is $417 \mathrm{~nm}$ ) [15].

\section{Gut permeability assays}

The gut permeability assays were performed at the end of 12-weeks diet intervention. The gavage volumes of FITC-conjugated dextran (supplier company is Sigma, USA) were calculated by body weight, the final concentration is $0.4 \mathrm{mg} / \mathrm{g}$ body weight. After 4 hours of gavage, plasma fluorescence at $485 / 528 \mathrm{~nm}$ was measurement according to standard samples, the machine model is Synergy H1 Bio-tek [15].

\section{Quantitative real-time PCR}

RNA samples were isolated using Qiazol reagent, the isolating protocol was established according to the Roche kit's instructions. Roche Transcriptor Universal cDNA Master kit was selected to synthesize cDNA from RNA samples according to their instructions (Roche, Mannheim, Germany). Quantitative real-time PCR was done using SYBR Green Master Mix reagent (Roche) with an Applied Biosystems amplification machine (the machine model is $7900 \mathrm{HT}$ ). To reduce error, each sample was run in triplicate and PPIA housekeeping gene were used to normalized the genes expression. Relative fold changes in gene expression normalized to itself's PPIA gene were then analyzed by the $\triangle \triangle C T$ method (the concrete equation is $2^{-\triangle \Delta C T}$ ). The results of calculation using above method are presented as fold changes relative to the transcript levels of the control. The following primers were used to verify the expression of the target genes: PPARY: forward, GCCCTTTGGTGACTTTATGG, reverse, CTCGATGGGCTTCACGTT; PPARa: forward, TTCCCTGTTTGTGGCTGCTAT, reverse, TGCAACTTCTCAATGTAGCCTATGTT; PPIA, forward, TTCCTCCTTTCACAGAATTATTCCA, reverse, CCGCCAGTGCCATTATGG [15].

\section{Statistical analysis}

Mean \pm standard error of the mean (SEM) were present to show the data obtained in study. For comparisons between two groups, Student's ttests was used for calculation. And for comparisons of 3 groups or more than 3 groups, one-way analysis of variance (ANOVA) was used for the statistical analyses. All analyses were performed using GraphPad Prism 5. To show the statistically significant results, $P<0.05$ was considered and noted in figures.

\section{RESULTS}

\section{5-ASA treatment did not affect metabolic basic parameters and IR-related factors in mice}

After a 12-week diet intervention, mice fed HFD or HFC diet gained much more body weight as well as body fat mass than those in LFD group (Figure 1A, B). However, mice fed LFD or HFD or HFC diet did not show significant differences in body composition (Figure 1B) and food intake (Figure 1C) when compared with the 5-ASA treated and non-treated groups.

In order to investigate whether 5-ASA improves systemic IR in mice fed HFD and to test if 5-ASA improves these parameters when mice are fed HFC diet, GTT was carried out after 11 weeks of dietary intervention. Compared to mice assigned to LFD group, the HFD and HFC groups displayed remarkably higher fasting blood glucose levels (Figure 2A) while performing GTT (Figure 2C). Surprisingly, contrary to previous reports, mice treated with 5-ASA showed similar fasting glucose (Figure 2A), fasting insulin (Figure 2B) and glucose tolerance (Figure 2C) irrespective of diet. These results suggest that 5ASA has no remedial effects on the metabolic and homeostasis of glucose in diet-induced obesity.

\section{5-ASA treatment improved lipid level in mice fed on HFC diet}

Although reduced liver steatosis showed by H\&E staining in the HFD 5-ASA group by Luck et al seemed rather attractive, their study did not involve quantitative measurement of plasma and hepatic lipid levels. Treatment with 5-ASA did not affect liver weight in mice fed LFD, HFD or HFC diet (Figure 3A). Results from histology showed no obvious differences in lipid accumulation between 5-ASA-treated mice and those that did not receive 5-ASA (result not shown). However, analysis of liver lipid profile showed a mild but significant reduction in triglycerides (Figure 3B), but not in total and free cholesterol (Figures $3 \mathrm{C}$ and Figure 3D) in the 5-ASA treated mice, when compared to HFC-fed mice without 5-ASA exposure. 
A

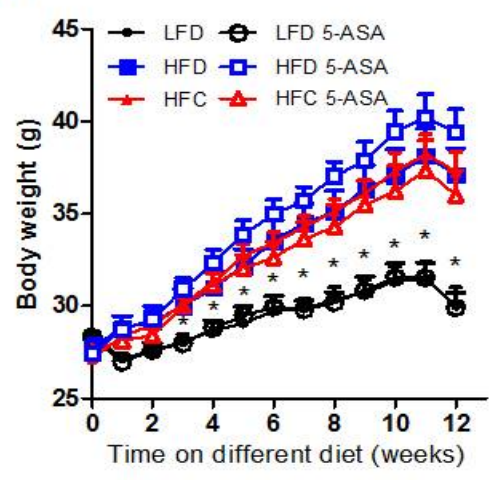

B

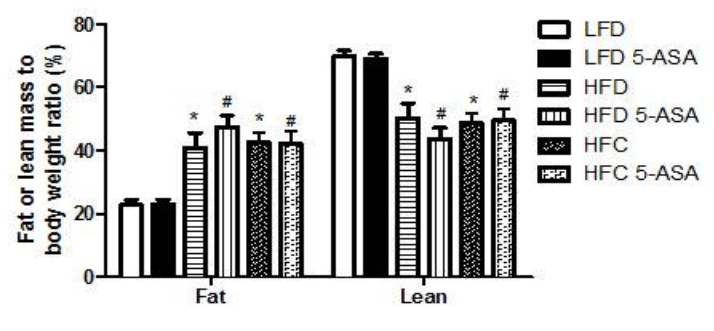

C

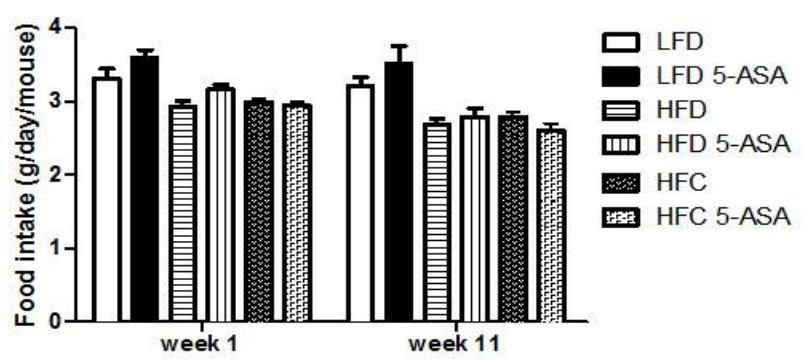

Figure 1: Effect of 5-ASA treatment on body composition and food intake of mice. Note: (A) Body weights of mice fed on different diets over time, ${ }^{*} p<0.05$ vs. LFD. (B) Fat or lean mass of mice after 12 weeks on different diets. ${ }^{*} p<0.05$ vs. LFD; \#p $<0.05$ vs. LFD $+5-A S A$. (C) Food intake of mice fed on different diets in the first week and $11^{\text {th }}$ week. Data obtained here are showed as mean \pm SEM, $n=9-10$ mice

A

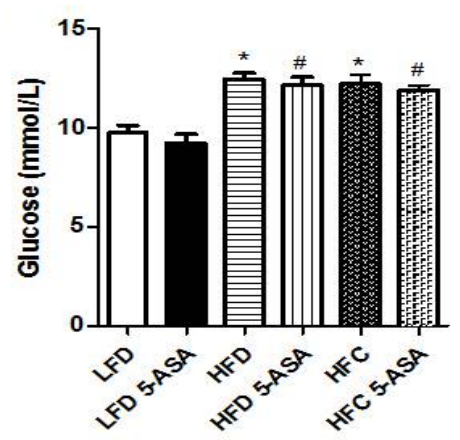

B

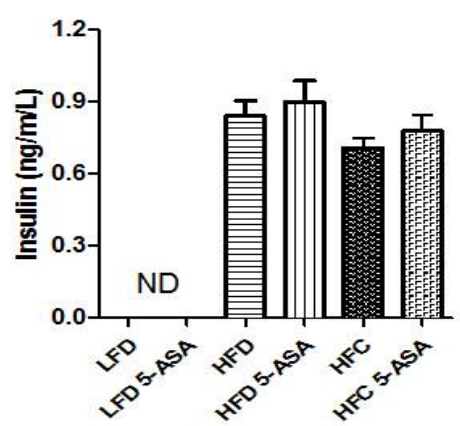

C

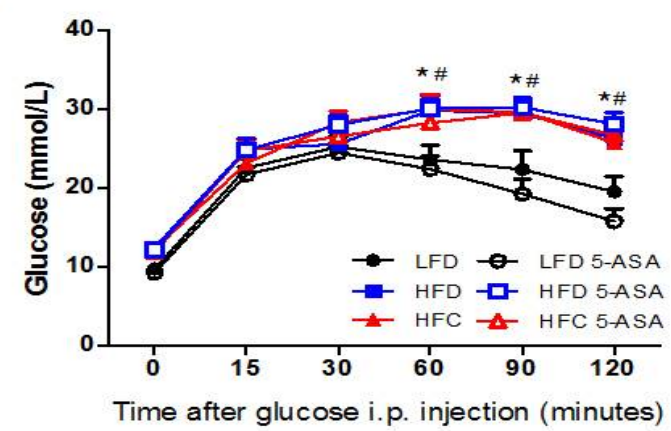

Figure 2: Effect of 5-ASA treatment on IR-related metabolic parameters in mice. Note: (A) Fasting glucose, (B) fasting insulin and (C) GTT of mice fed different diets for 12 weeks. ${ }^{*} p<0.05$ vs. LFD; \#p $<0.05$ vs. LFD 5-ASA. Data obtained here are showed as mean \pm SEM, $n=9-10$ mice 
Relative to LFD groups, HFD or HFC exposure for 12 weeks did not affect plasma triglycerides (Figure 3E), but it significantly elevated plasma total cholesterol and free cholesterol (Figure $3 F$ and Figure G). In HFD and HFC groups, 5-ASA treatment led to a downward trend in plasma total cholesterol and free cholesterol (Figure 3F and Figure G). Interestingly, mice fed on LFD + 5-ASA showed lower triglyceride level than those fed LFD $(p=0.052)$. Mice in the HFC 5-ASA group had significantly attenuated plasma triglyceride when compared to the HFC group (Figure 3E). However, cholesterol levels were not affected by 5-ASA in any of the diets (Fig. 3F and $G)$. These data suggest that 5-ASA may act as a lipid regulator especially when mice are challenged with a HFC diet.

\section{Effect of 5-ASA on small intestine}

There were no traces of 5-ASA and its metabolites in circulation (Figure 4A). This confirms the functionalization of 5-ASA in small intestine through the up regulation of mRNA expression of its target gene, which is also well accepted to be the anti-inflammatory target, peroxisome proliferator-activated receptor-Y (PPARy, Figure 4B). After 3 weeks of treatment, 5 -ASA induced significant changes in gut microbial flora when compared to HFD and HFC diet (data not shown). However, results of fluorescence FITC-dextran 4 (FD4) assay showed that 5-ASA treatments did not improve intestinal epithelial barrier permeability (Figure 4C) irrespective of diet used. This is consistent with the results related to glucose homeostasis.

Since PPARs (including PPARY and PPARa) are generally considered as master regulators of lipid homeostasis, studies were carried out to determine whether the lipid lowering effect of 5ASA was associated with PPARa. The results revealed that the expression of PPARa mRNA in small intestine in mice fed HFC + 5-ASA was significantly higher than in mice fed HFC only (Figure 4D). Similar results were seen for the downstream target genes of PPARa, such as HSL, AOX and CTP1a, which play roles in lipolysis and fatty acid oxidation (data not shown).
A

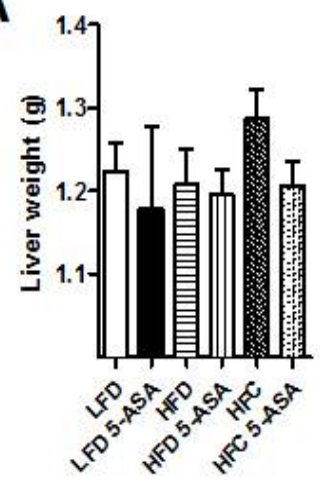

B

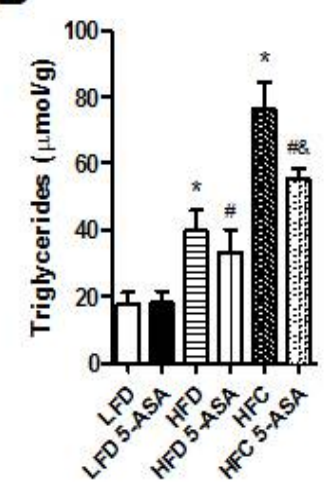

F
C

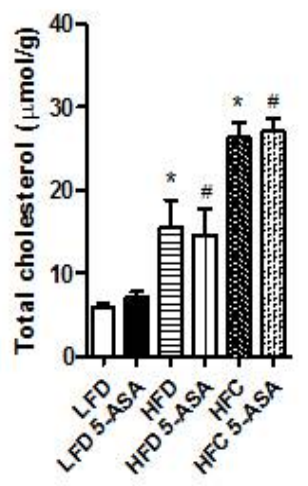

G
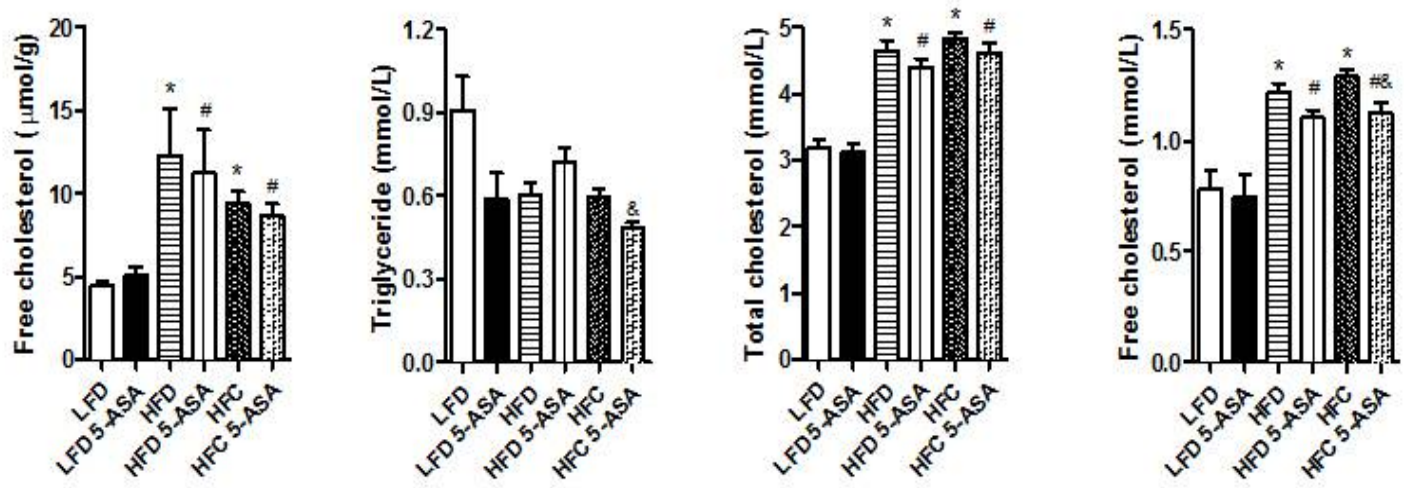

Figure 3: Effect of 5-ASA on lipid profile in mice fed either LFD, HFD or HFC diet. Note: (A) Liver weight of mice after 12 weeks feeding of different diets. (B-D) Liver triglycerides, total cholesterol and free cholesterol concentrations of mice after 12 weeks dietary. (E-G). * $p<0.05$ vs. LFD; \#p<0.05 vs. LFD+5-ASA; \& $p<0.05$ vs. $H F C+5-A S A$. Data obtained here are showed as mean $\pm S E M, n=9$ to 10 mice 
A

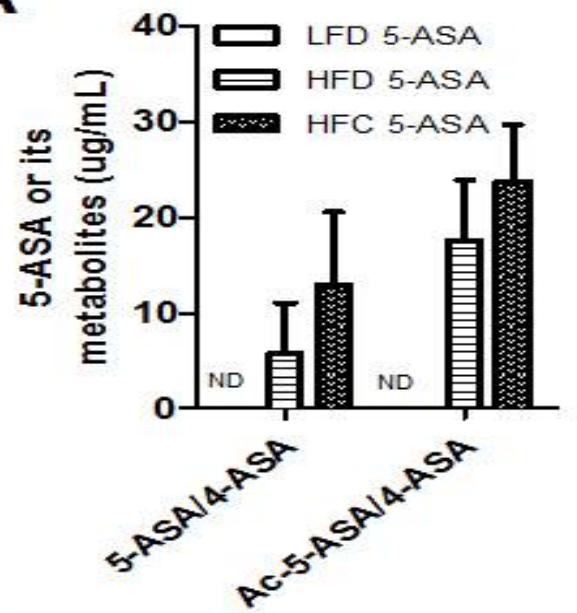

C

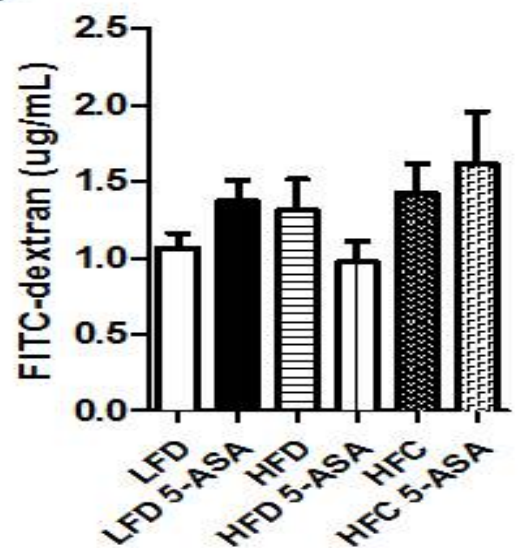

$\mathbf{B}$

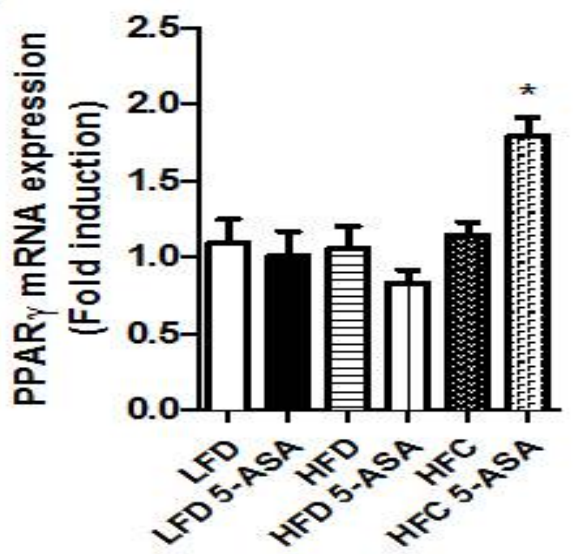

$\mathbf{D}$

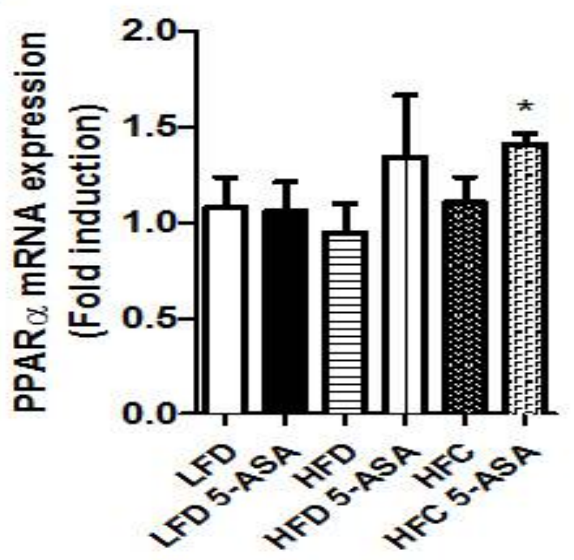

Figure 4: Effect of 5-ASA on small intestine. Note: (A) 5-ASA and its metabolites concentration in plasma after 3 weeks of diet intervention. Values are mean \pm SEM, $n=4-5$ mice. (B) PPARY mRNA expression relative to PPIA, which is the housekeeping gene expression in small intestine of mice after 12 weeks dietary intervention; ${ }^{*} p<$ 0.05 vs. HFC. (C) Plasma FD4 levels, measured at 4 hours after oral gavage of mice at the end of 12 weeks of diet intervention. (D) PPAR $m R N A$ expression relative to PPIA, which is the housekeeping gene expression in small intestine of mice at the end of 12 weeks dietary intervention; ${ }^{*} p<0.05 \mathrm{vs}$. HFC

\section{DISCUSSION}

Improved understanding claimed obesity-related complications are characterized and advanced by chronic low-grade inflammation, and target inflammation may block the development of chronic metabolic syndromes such as IR and dyslipidemia [17-19]. Although anti-inflammatory agents show some beneficial outcomes in animal studies and clinical trials $[4,5,20]$. Systemic antiinflammatory therapies are not ideal since inflammatory response is rather critical for host defense. Moreover, anti-inflammation may lead to serious side effects. Indeed, studies from animal and human suggest inflammation in obesity is not always deleterious, while results from many studies are controversial [6].

Recently, people proved HFD induced gut inflammation and then triggers metabolic disorders while anti-enteritis agent, 5-ASA reverses IR through improvement of fasting blood glucose, insulin levels and glucose tolerance [21]. These are at variance with the data acquired in this study, which showed that 5ASA treatment did not have a specific effect on fasting blood glucose levels, fasting plasma insulin levels and GTT response in mice fed either LFD, HFD or HFC. This finding indicates that associations between gut anti-inflammatory agent and IR should be interpreted with caution.

Additionally, HFD+5-ASA-fed mice showed reduced liver steatosis when compared to HFD [9]. A similar downtrend in hepatic and plasma lipid levels in mice given 5-ASA+HFD, especially in mice fed HFC. 5-ASA shares many of the pharmacological properties of non-steroidal antiinflammatory drugs (NSAIDs), for instance aspirin. Intake of aspirin resulted in decreased serum total cholesterol and triglycerides in patients [22] while chronic administration of 
NSAIDs is effective in preventing steatohepatitis and atherosclerosis in mice fed HFD [11]. These findings reveal the function of systemic and intestinal inflammation in the pathogenesis and progress of diet-induced dyslipidemia. However, the results showed here indicated that the lipidmodulatory effect of 5-ASA may be independent of its effect on IR.

5-ASA is widely used in the therapeutic and maintenance treatment of IBD and is among the oldest anti-inflammatory agents in use today. Although the exact mechanisms of action of 5ASA are not completely elucidated, recent studies have revealed that multiple intestinal antiinflammatory effects of 5-ASA rely on increased expression of PPARy; As a receptor of 5-ASA [23,24], PPARy and PPARa not only act effectively as anti-inflammatory targets, but also a major lipid sensors that control the expression of genes related to lipid and carbohydrate metabolism [25]. The present study reports that the lipid-lowering effect of 5-ASA is associated with enhanced PPARs and the function of their downstream target genes in fatty acid oxidation in the small intestine for the first time and more experiment was urgent needed to further elucidate the mechanism.

\section{CONCLUSION}

The present study explored the role of gut inflammation in the pathogenesis of diet-induced metabolism disorders. The findings show that targeting intestinal inflammation does not result in obvious improvement of IR parameters. However, 5-ASA treatment is associated with a lipid-lowering effect in mice fed HFC diet, and this effect is related to the activation of PPARs in gut. Overall, it is rather promising that gut inflammation may serve as a distinct target in control diet-induced dyslipidemia.

\section{DECLARATIONS}

\section{Acknowledgement}

WZ and BZJ were supported by a grant from Shanghai Municipal Commission of Health and Family planning, Shanghai Key Developing Disciplines Program (no. 2015ZB0501). The authors thank Marten Hofker's team for their technical guidance and comments for preparing the manuscript.

\section{Conflict of Interest}

No conflict of interest associated with this work.

\section{Contribution of Authors}

The authors declare that this work was done by the authors named in this article and all liabilities pertaining to claims relating to the content of this article will be borne by them.

\section{Open Access}

This is an Open Access article that uses a funding model which does not charge readers or their institutions for access and distributed under the terms of the Creative Commons Attribution License (http://creativecommons.org/licenses/by/ 4.0) and the Budapest Open Access Initiative (http://www.budapestopenaccessinitiative.org/rea d), which permit unrestricted use, distribution, and reproduction in any medium, provided the original work is properly credited.

\section{REFERENCES}

1. Saltiel AR, Olefsky JM. Inflammatory mechanisms linking obesity and metabolic disease. J Clin Investig 2017; 127(1): 1-3.

2. Ding S, Lund PK. Role of intestinal inflammation as an early event in obesity and insulin resistance. Curr Opin Clin Nutr Metab Care 2011; 14(4): 328-330.

3. Megna BW, Carney PR, Kennedy GD. Intestinal inflammation and the diet: Is food friend or foe? World $J$ Gastrointest Surg. 2016; 8(2): 115-123.

4. Donath MY. Targeting inflammation in the treatment of type 2 diabetes. Diabetes Obesity \& Metabolism 2013; 15(s3): 193-196.

5. Goldfine $A B$, Shoelson SE. Therapeutic approaches targeting inflammation for diabetes and associated cardiovascular risk. Jo Clin Investig. 2017; 127(1): 8385.

6. Ye J, Mcguinness OP. Inflammation during obesity is not all bad: evidence from animal and human studies. Am J Physiology Endocrinol Metab. 2013; 304(5): E466-E477.

7. Ding SL, Chi MM, Scull BP, Rigby R, Schwerbrock NMJ, Magness S, Jobin C, Lund PK. High-fat diet: bacteria interactions promote intestinal inflammation which precedes and correlates with obesity and insulin resistance in mouse. Plos One 2010; 5(8): e12191.

8. Wit NJD, Boschvermeulen H, Groot PJD, Hooiveld GJ, Bromhaar MMG, Jansen J, Müller M, Meer RVD. The role of the small intestine in the development of dietary fat-induced obesity and insulin resistance in C57BL/6J mice. BMC Med Genomics 2008; 1(1): 1-16.

9. Luck $H$, Tsai $S$, Chung J, Clemente-Casares $X$, Ghazarian M, Revelo XS, Lei H, Luk CT, Shi SY, Surendra $A$. Regulation of Obesity-Related Insulin Resistance with Gut Anti-inflammatory Agents. Cell Metabolism 2015; 21(4): 527-529. 
10. Rozemond $H$. The principles and guidelines established by the European Convention for the Protection of Laboratory Animals. Vet Q 1986; 8(4):346-349.

11. Madrigalperez VM, Garcíarivera A, Rodriguezhernandez A, Cejaespiritu G, Briseñogomez XG, Galvansalazar $H R$, Sorianohernandez $A D$, Guzmanesquivel J, Martinezfierro ML, Newtonsanchez OA. Preclinical analysis of nonsteroidal anti-inflammatory drug usefulness for the simultaneous prevention of steatohepatitis, atherosclerosis and hyperlipidemia. Int $\mathrm{J}$ Clin Exp Med. 2015; 8(12): 22477-22479.

12. Progatzky F, Sangha NJ, Yoshida N, Mcbrien M, Cheung $J$, Shia A, Scott J, Marchesi JR, Lamb JR, Bugeon L. Dietary cholesterol directly induces acute inflammasome-dependent intestinal inflammation. Nat Communications 2014; 5(5): 5864-5867.

13. Rousseaux C, Lefebvre B, Dubuquoy L, Lefebvre $P$, Romano O, Auwerx J, Metzger D, Wahli W, Desvergne $B$, Naccari GC. Intestinal antiinflammatory effect of 5 aminosalicylic acid is dependent on peroxisome proliferator-activated receptor-gamma. J Exp Med. 2005; 201(8): 1205-1215.

14. Dubuquoy L, Rousseaux $C$, Thuru $X$, Peyrin LB, Romano $O$, Chavatte $P$, Chamaillard $M$, Desreumaux $P$. PPAR\{gamma\} as a new therapeutic target in inflammatory bowel diseases. Gut 2006; 55(9): 1341 1349.

15. Wahli W, Michalik L. PPARs at the crossroads of lipid signaling and inflammation. Trends Endocrinol. Metab Tem 2012; 23(7): 351-354.

16. Organization. WH. Principles of laboratory animal care. WHO Chron 1985; 39: 51-56.

17. Winer S, Yin C, Paltser G, Truong D, Tsui H, Bahrami J, Dorfman R, Wang $Y$, Zielenski J, Mastronardi F.
Normalization of obesity-associated insulin resistance through immunotherapy. Nat Med. 2009; 15(8): 921 929.

18. Bligh EG, Dyer WJ. A rapid method of total lipid extraction and purification. Can J Biochem Physiol. 1959; 37(8): 911-915.

19. Dong CX, Zhao W, Solomon C, Rowland KJ, Ackerley C, Robine S, Holzenberger M, Gonska T, Brubaker PL. The intestinal epithelial insulin-like growth factor-1 receptor links glucagon-like peptide-2 action to gut barrier function. Endocrinology 2014; 155(2): 370-372.

20. Tabas I, Glass CK. Anti-Inflammatory Therapy in Chronic Disease: Challenges and Opportunities. Sci. 2013; 339(6116): 166-172.

21. Li H, Lelliott $C$, Hakansson $P$, et al. Intestinal, adipose, and liver inflammation in diet-induced obese mice. Metabolism 2008; 57(12): 1704-1710.

22. van Diepen JA, Berbée JF, Havekes LM, Rensen PC. Interactions between inflammation and lipid metabolism: Relevance for efficacy of anti-inflammatory drugs in the treatment of atherosclerosis. Atherosclerosis 2013; 228(2): 306-315.

23. Ohashi K, Shibata R, Murohara T, Ouchi N. Role of antiinflammatory adipokines in obesity-related diseases. Trends in Endocrinol Metab Tem. 2014; 25(7): 348-350.

24. Panahi $Y$, Dadjo Y, Pishgoo B, Akbari A, Sahebkar A. Clinical evaluation of the anti-inflammatory effects of Heracleum persicum fruits. Comparative Clinical Pathology 2015; 24(4): 971-974.

25. Hundal RS, Petersen KF, Mayerson AB, Randhawa PS, Inzucchi S, Shoelson SE, Shulman Gl. Mechanism by which high-dose aspirin improves glucose metabolism in type 2 diabetes. J Clin Investig. 2002; 109(10): 1321 1324. 